\title{
A Case of Lichen Sclerosus et Atrophicus Accompanying Bullous Morphea
}

\author{
Sirin Yasar, M.D., Ceyda Tanzer Mumcuoglu, M.D., Zehra Asiran Serdar, M.D., \\ Pembegul Gunes, M.D. ${ }^{1}$ \\ Departments of Dermatology, ${ }^{1}$ Pathology, Haydarpaş Numune Training and Research Hospital, Istansbul, Turkey
}

Bullous morphea is a rare form of morphea characterized with bullae on or around atrophic morphea plaques. Whereas lichen sclerosus et atrophicus (LSA) is a disease the etiology of which is not fully known, and which is characterized with sclerosis. Coexistence of morphea and LSA has been identified in some cases. Some authors believe that these two diseases are different manifestations which are on the same spectrum. The 70-year-old patient stated herein, presented to us for 6 months with annular, atrophic plaques, ivory color in the middle, surrounded by living erythema, on the front and back of the trunk. Occasionally bulla formation on the plaques on the trunk lateral was identified. Fibrotic and atrophic plaques of ligneous hardness were present on the front side of tibia of both legs. In the histopathologic examination, the lesions were found concordant with bullous morphea and LSA. With colchicine $1.5 \mathrm{mg} /$ day, pentoxifylline $1,200 \mathrm{mg} /$ day, topical calcipotriol ointment and clobetasol propionate cream, the erythema in the patient's lesions faded and softening in the fibrotic plaques was observed. Concomitance of bullous morphea and LSA is a rarely seen, interesting coexistence which suggests a common, as yet unknown, underlying pathogenesis. (Ann Dermatol 23(S3) S354 S359, 2011)

Received April 14, 2010, Revised May 8, 2011, Accepted for publication May 9, 2011

Corresponding author: Ceyda Tanzer Mumcuoglu, M.D., Haydarpaşa Numune Eğitim ve Araştırma Hastanesi Dermatoloji Kliniği Üsküdar, İstanbul, Turkey [34746]. Tel: 902164144502, Fax: 902164144502, E-mail: ceydatanzer@gmail.com

This is an Open Access article distributed under the terms of the Creative Commons Attribution Non-Commercial License (http:// creativecommons.org/licenses/by-nc/3.0) which permits unrestricted non-commercial use, distribution, and reproduction in any medium, provided the original work is properly cited.
-Keywords-

Lichen sclerosus et atroficus, Scleroderma, localised

\section{INTRODUCTION}

Bullous morphea is a rare form of morphea characterized with bullae on or around atrophic morphea plaques, as defined for the first time by Morrow in $1959^{1}$.

Lichen sclerosus (LS) is a chronic inflammatory skin disease, which most commonly involves the anogenital region. The etiology of LS is obscure, but genetic susceptibility, autoimmune mechanisms, infective agents such as human papilloma virus and spirochaetes, as well as Koebner phenomenon have been postulated as causative factors ${ }^{2}$.

Coexistence of morphea and lichen sclerosus et atrophicus (LSA) has been identified in some cases $^{2-11}$. Some authors believe that these two diseases are different clinical manifestations on the same spectrum. Edema, paling, and collagen homogenization in the papillary dermis are more prominent in LSA; whereas the reticular dermis is also affected in morphea, and coarsening and parallel skin arrangement of collagen and atrophy in skin appendages is also evident. A 70-year-old male patient presented herewith, whose simultaneously occurring lesions for 6 months were found to be concordant with bullous morphea and LSA. Concomitance of bullous morphea and LSA is an interesting and rarely seen occurrence, and has a common and yet unexplained underlying pathogenesis.

\section{CASE REPORT}

A 70-year-old male patient presented with complaints of wounds that were red around the edges, hard in the middle, and occasionally turned to blisters on the front 

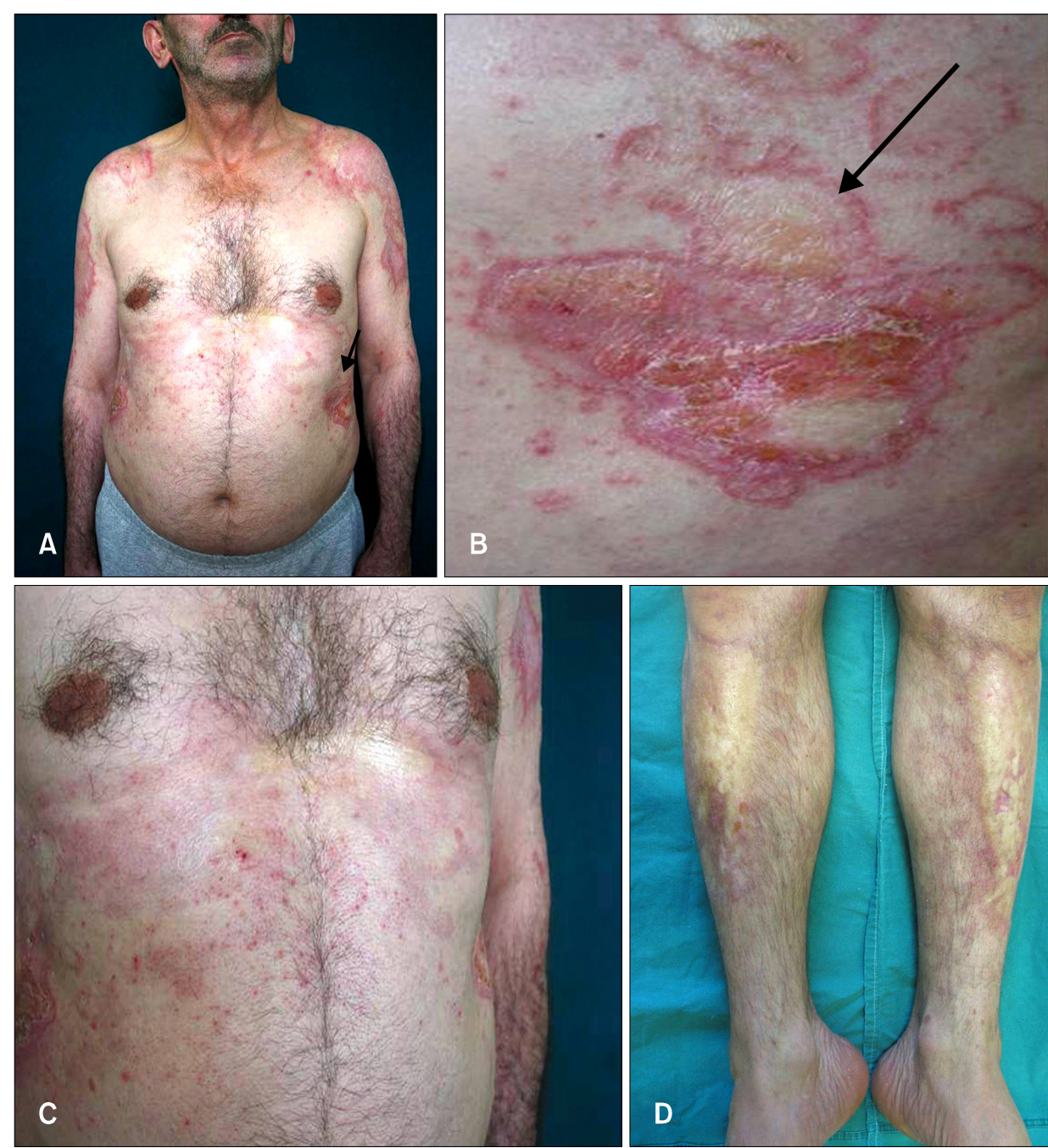

Fig. 1. $(A \sim C)$ Circinate, atrophic plaques surrounded by living erythema. Indurated plaques, whcih are bullous in the middle. (D) Fibrotic plaques on the anterior aspect and surface of the tibia. and back of the trunk, ligneous hardening of the legs, and restriction and pain in knee joints for 6 months. He had occasionally applied topical creams to these aliments, but had not seen any benefit. He had a history of smoking 55 packs/year for many years and loss of hearing in the left ear. No characteristics were identified in his family history. A system investigation and physical examination were within normal limits. The dermatological examination revealed:

* Circinate, atrophic plaques, surrounded by living erythema, yellow in the middle, ivory colored in places, in the form of a mane on both shoulders and in the mid-line on the back (Fig. 1A, B)

* Indurated plaques on the abdomen, which were bullous in the middle (Fig. 1C)

* Fibrotic plaques surrounded bilaterally with living erythema, which were hard in the middle, and located on the anterior aspect or surface of the tibia (Fig. 1D).

The laboratory findings were positive C3 anti-HBs (hepatit
B virus surface anticore) of $164 \mathrm{mg} / \mathrm{dl}$ (normal range, 79 152), positive (1:120 titer) antinuclear antibodies (ANAs) by indirect immunofluorescence (Hep-2 cells) (homogenous pattern), and negative anti-single-stranded DNA, anti double-stranded DNA, anti-SSA/Ro and Scl-70 but positive anti-SSB/La. Evaluations were made for the Lyme's disease Ig $M$ antibody (EIA) was negative, but the Lyme Ig G antibody (EIA) was positive at the detection limit (16 $\mathrm{RU} / \mathrm{ml})$.

Increased bilateral interstitial density was detected on pulmonary radiography; and moderate restrictive and obstructive combined type respiratory failure was observed on respiratory function tests. Thoracic computed tomography $(\mathrm{CT})$ revealed changes concordant with bullous and fibrotic tissue in subpleural areas, which were more prominent in the upper lobe apex of the right lung. Density changes were evident in the basal lower lobe of both lungs concordant with a chronic interstitial retention. High resolution CT was performed; however, no pathol- 


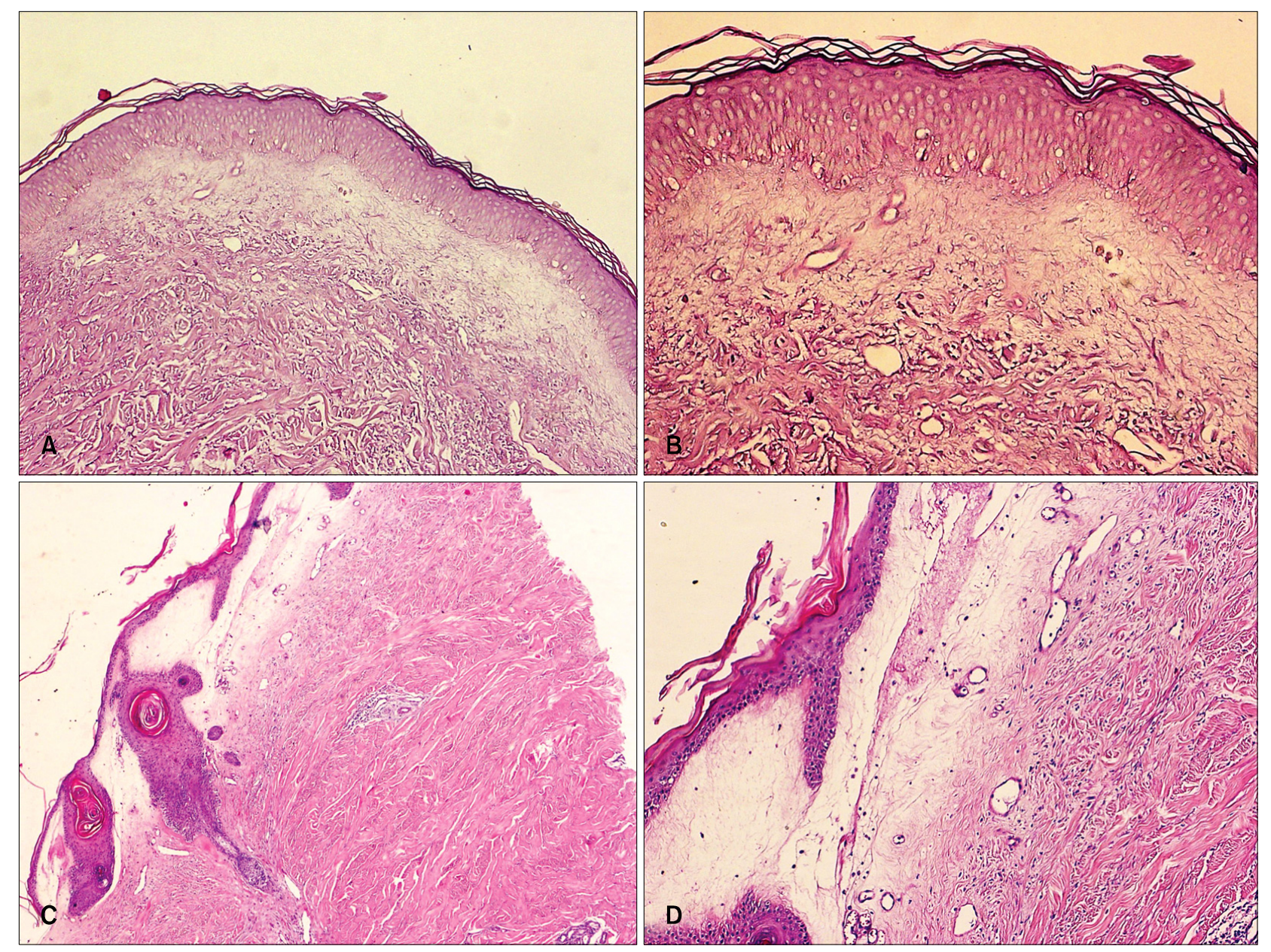

Fig. 2. (A, B) Epidermal atrophy and flattening of the rete ridges, vacuolar degeneration in the basal layer, edema in the papillary dermis $(H \& E, \times 40,100)$. (C, D) Subepidermal detachment in a focal area, coarsened collagen fibers $(H \& E, \times 40,100)$.

ogy was found. Ultrasonography performed on the legs revealed that skin thickness had increased due to fibrosis. The histopathological examination performed on the atrophic follicular lesions on the upper back showed basketweave hyperkeratosis in the epidermis, atrophy and flattening of the rete ridges, vacuolar degeneration in the basal layer, edema in the papillary dermis, a pale appearance, homogenization, melanine incontinence. Perivascular lymphocyte infiltration was observed on the skin appendages (Fig. 2A, B). These findings were believed to consistent with a histological diagnosis of LSA. Atrophy in the epidermis, deletion and flattening in the retes, and subepidermal disintegration in the focal area were observed on the biopsy performed on the atrophic area on which bulla had developed on the abdomen. The papillary dermis was severely edematous. Coarsening of collagen fibers, the presence of a parallel to surface arrangement, and paling were observed in the mid and reticular dermis. In most areas, skin appendages were re- moved by coarse collagen fibers, whereas intensive lymphocyte infiltration was present in the reticular dermis (Fig. 2C, D). The findings were believed to be characteristic of morphea.

Additionally, we performed Masson's trichrome and elastic Von Gieson staining to evaluate the collagen and elastic fibers. Collagen bundles were sclerotic and coarseness and elastic fibers were present within the sclerosing areas the areas with morphea. Collagen bundles were not sclerotic and the coarseness and elastic fibers decreased markedly in the dermis of areas with LSA (Fig. $3,4)$.

As a result, the patient was diagnosed with LSA and bullous morphea. An iommunofluorescence test and laser scanning confocal microscopy were not performed.

Erythema of the lesions faded and the fibrotic plaques softened 6 weeks later after treatment with $1.5 \mathrm{mg} /$ day colchicine, 1,200 mg/day pentoxifylline, topical calcipotriol, and clobetasol propionate cream. As the patient 

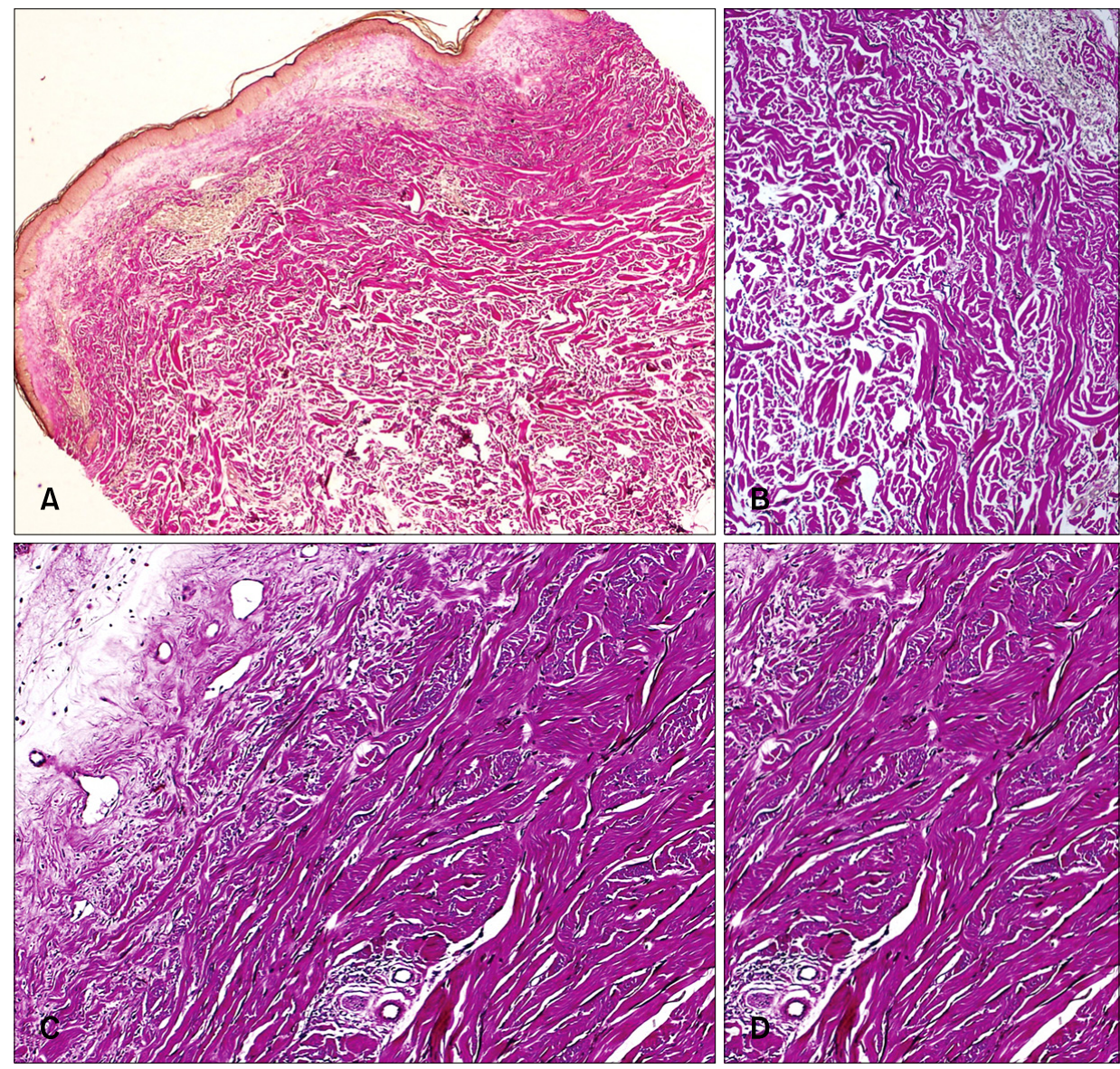

Fig. 3. (A, B) Decrease of elastic fibers in lichen sclerosus et atrophicus (elastic von Gieson, $\times 40$, 100), (C, D) Normal limits of elastic fibers in morphea (elastic von Gieson, $\times 40,100)$.

did not attend follow-up visits regularly, information could not be obtained on progress.

\section{DISCUSSION}

Bullous morphea is a rarely observed form of morphea. The bullous lesions are often localized to the lower abdominal area and lower extremities.

The bulla development mechanism in the sclerotic area is unclear. However, various hypotheses may explain bulla formation. Templeton claimed that bulla are caused by a lymphatic obstruction, and that the presence of enlarged lymphatic channels in the upper dermis supported this claim $^{2}$. Additionally, due to increased hydrostatic pressure, bullous lesions are more frequently observed on the lower extremities. Other authors believe that bulla is caused by nerve damage from trauma, deposition of eosinophilic granule major basic protein, or enzymatic damage in the basal membrane caused by a vascular occlusion $^{3,12}$. However, in our case, the histopathological examination did not reveal increased eosinophils in tissue or serum or any vascular changes or lymphatic dilatation obstruction that would explain the pathogenesis.
The correlation between spirochetes and bullous morphea is disputable. In our case, evaluations were made for the Lyme Ig $M$ antibody, which was negative, and the Lyme Ig $\mathrm{G}$ antibody, which was positive at the detection limit (16 RU/ml); however, the results did not suggest a Borrelia burgdorferi infection.

LSA is a chronic disease of the skin and mucosae, also known as kraurosis vulvae or balanitis xerotica obliterans ${ }^{13}$. Despite being mostly localized to the anogenital region, it may also be localized on the upper parts of the back and chest area. Although B. burgdorferi, an autoimmune disorder, or trauma are major causes for this disease, the exact reason is unknown. Histopathologically distinguishing LSA from morphea in the extragenital region is difficult and sometimes even impossible. Lever stated that a classic LSA lesion shows hyperkeratosis with follicular plugging, atrophy of the Malpighian layer with vacuolar degeneration of the basal layer, edema. and homogenization of the collagen in the upper dermis, as well as an inflammatory infiltrate in the mid-dermis. However, all these findings are not always present, which makes interpretation of the histological diagnosis more difficult ${ }^{14}$. Our findings agreed with those of LSA, except for the follicular 

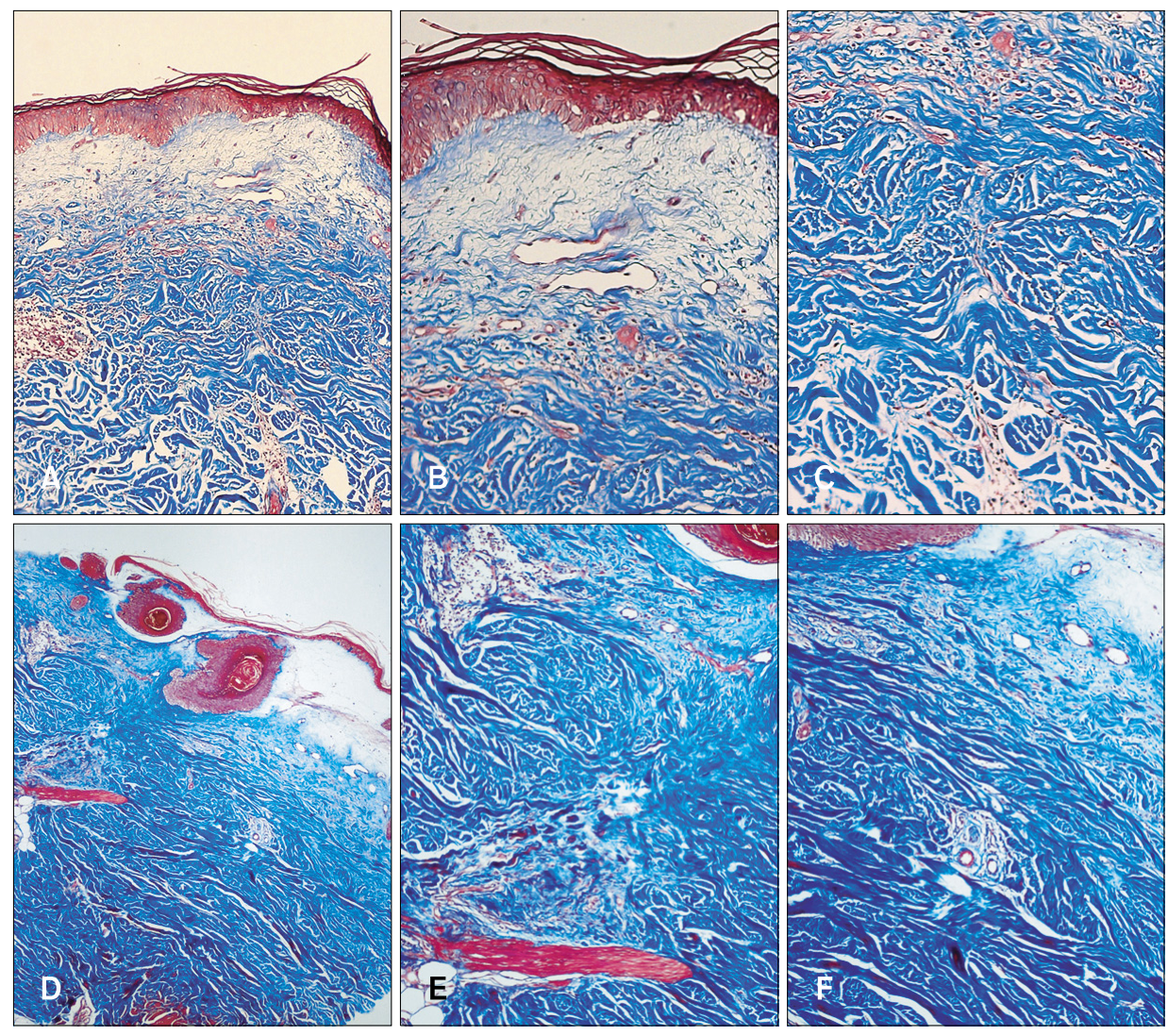

Fig. 4. $(A \sim C)$ In lichen sclerosus et atrophicus, normal collagen fibers (Masson's trichrome, $\times 40,100,200)$, $(D \sim F)$ coarse, sclerosing collagen fibers in morphea (Masson's trichrome, $\times 40,100,200$ ).

plugging.

Borda et al. considered LSA as scleroderma of the papillary dermis and claimed that it could be a form of scleroderma. Uitto et al. also thought that histological findings of LSA and morphea could coexist in the same lesion, indicating that the two clinical lesions may, in some instances, represent a spectrum of disease rather than separate entities $^{11}$. Connelly and Winkelmann argued that lichen slerosus and morphea are a spectrum of the same disease and that LSA is subepidermal morphea ${ }^{15}$. Patterson and Ackerman believe that morphea and LSA are different clinical manifestations of the same disease spectrum. While changes in the papillary dermis are prominent in LSA, the reticular dermis is affected and concomitant systemic disease increases in patients with morphea ${ }^{16}$. Several investigators have suggested additional histological techniques to distinguish between these two conditions when there is some uncertainty about the diagnosis. These include elastin staining (absent or greatly reduced in the upper dermis of LSA but not in morphea) $)^{17,18}$ and differences in the dermal ground substance. Kowalewski et al. investigated alterations in the basement membrane zone (BMZ) and cutaneous microvasculature in patients with morphea and extragenital LSA using laser scanning confocal microscopy. They showed a decreased number

and size of dermal papillae both in patients with morphea and LSA compared with those in normal skin. In patients with morphea, continuity of the $B M Z$ at the level of lamina lucida, lamina densa and sublamina densa are preserved with numerous invaginations and holes in the $\mathrm{BMZ}$ at the level of the lamina lucida and lamina densa. They thought that alterations in the BMZ in patients with morphea differed from those in patients with $\mathrm{LSA}^{19}$.

The coexistence of morphea and LSA has been identified in some cases. It is unclear why bullous morphea and LSA coexist; however, both diseases have some common pathogenetic characteristics. The fact that spirochetes are involved in the etiologies of both diseases also supports a common pathogenesis. This close relationship raises the question of whether morphea and LSA are different indications of the same disease. Morphea and LSA have been associated with autoimmune diseases and autoantibodies of various types. Perhaps common autoimmune phenomena, a genetic predisposition, or a specific reaction to infection may play a role in patients with morphea-LSA. Anti-SSB/La and ANAs were detected in our patient, supporting the autoimmune hypothesis. Additionally, Dickie et al. provided direct evidence that LSA may have an immunological basis by showing positive immunofluorescent (IF) staining with fibrin, C3, or IgM in 
the basement membrane of lesional $\operatorname{skin}^{20}$. But, we did not perform IF staining due to it unavailability in our hospital.

Coexistence of bullous morphea and LSA has been reported in one case in the literature; however, that patient also had systemic lupus erythematosus ${ }^{3}$. No underlying spirochete infection was observed in our patient, nor any other autoimmune disease or history of trauma. The coexistence may have been coincidental; however, common histopathological aspects such as intense edema and paling in the papillary dermis suggest the existence of the same disease in different developmental stages. We thought that the patient's diagnosis was bullous morphea due to decreases in the normal limits of elastic fiber, sclerosing collagen, and diminished skin appendages but made the LSA diagnosis because of the decrease in elastic fibers and the absence of sclerosing collagen.

Corticosteroids, antimalarial drugs, colchicine, retinoids, salazopyrin, pentoxifylline, UVA1 and wide band UVA are useful for treating bullous morphea. In our case, we achieved good results with $1.5 \mathrm{mg} /$ day colchicine, 1,200 $\mathrm{mg} /$ day pentoxifylline, topical calcipotriol ointment and clobetasol propionate cream.

The coexistence of bullous morphea and LSA is a rare condition and with an increase in the number of cases in the future, the question as to whether these two diseases are variants of the same disease will be clarified.

\section{REFERENCES}

1. Jia $H$, Chen XH, Shi JH, Zhao CX, Cao YH, Zeng XS, et al. A case of bullous morphea reported in mainland China. Int J Dermatol 2002;41:949-950.

2. Gupta S, Malhotra AK, Ajith C. Lichen sclerosus: role of occlusion of the genital skin in the pathogenesis. Indian J Dermatol Venereol Leprol 2010;76:56-58.

3. Wu KH, Dai YS, Tsai MJ, Lin SC, Wang LH, Huang MT, et al. Lichen sclerosus et atrophicus, bullous morphea, and systemic lupus erythematosus: a case report. J Microbiol Immunol Infect 2000;33:53-56.

4. Kim DH, Lee KR, Kim TY, Yoon MS. Coexistence of lichen sclerosus with morphoea showing bilateral symmetry. Clin Exp Dermatol 2009;34:e416-418.

5. Sawamura D, Yaguchi T, Hashimoto I, Nomura K, Konta R, Umeki K. Coexistence of generalized morphea with hisotological changes in lichen sclerosus et atrophicus and lichen planus. J Dermatol 1998;25:409-411.

6. Tremaine R, Adam JE, Orizaga M. Morphea coexisting with lichen sclerosus et atrophicus. Int J Dermatol 1990;29:486489.

7. Farrell AM, Marren PM, Wojnarowska F. Genital lichen sclerosus associated with morphoea or systemic sclerosis: clinical and HLA characteristics. Br J Dermatol 2000;143: 598-603.

8. Forschner A, Metzler G, Rassner G, Fierlbeck G. Morphea with features of lichen sclerosus et atrophicus at the site of a herpes zoster scar: another case of an isotopic response. Int J Dermatol 2005;44:524-525.

9. Virdi SK, Kanwar AJ. Generalized morphea, lichen sclerosis et atrophicus associated with oral submucosal fibrosis in an adult male. Indian J Dermatol Venereol Leprol 2009;75:56-59.

10. Blaya B, Gardeazabal J, de Lagrán ZM, Díaz-Pérez JL. Patient with generalized guttate morphea and lichen sclerosus et atrophicus. Actas Dermosifiliogr 2008;99:808-811.

11. Uitto J, Santa Cruz DJ, Bauer EA, Eisen AZ. Morphea and lichen sclerosus et atrophicus. Clinical and histopathologic studies in patients with combined features. J Am Acad Dermatol 1980;3:271-279.

12. Daoud MS, Su WP, Leiferman KM, Perniciaro C. Bullous morphea: clinical, pathologic, and immunopathologic evaluation of thirteen cases. J Am Acad Dermatol 1994;30:937943.

13. Odom RB, James WD, Berger TG. Lichen planus and related conditions. Andrew's diseases of the skin clinical dermatology. 9th ed. Philadelphia: WB Saunders, 2000:266-283.

14. Lever WF, Schaumburg-Lever G. Histopathology of the skin. 6th ed. Philadelphia: JB Lippincott, 1983.

15. Connelly MG, Winkelmann RK. Coexistence of lichen sclerosus, morphea, and lichen planus. Report of four cases and review of the literature. J Am Acad Dermatol 1985;12: 844-851.

16. Winer $\mathrm{LH}$. Elastic fibers in unusual dermatoses. AMA Arch Derm 1955;71:338-348.

17. Rahbari H. Histochemical differentiation of localized morphea-scleroderma and lichen sclerosus et atrophicus. J Cutan Pathol 1989; 16:342-347.

18. Patterson JA, Ackerman AB. Lichen sclerosus et atrophicus is not related to morphea. A clinical and histologic study of 24 patients in whom both conditions were reputed to be present simultaneously. Am J Dermatopathol 1984;6:323-335.

19. Kowalewski C, Kozłowska A, Górska M, Woźniak K, Krajewski $M$, Błaszczyk $M$, et al. Alterations of basement membrane zone and cutaneous microvasculature in morphea and extragenital lichen sclerosus. Am J Dermatopathol 2005; 27:489-496.

20. Dickie RJ, Horne $\mathrm{CH}$, Sutherland HW, Bewsher PD, Stankler L. Direct evidence of localised immunological damage in vulvar lichen sclerosus et atrophicus. J Clin Pathol 1982; 35:1395-1397. 